\title{
Effects of intraocular pressure and aspheric transition zone ablation profile on corneal biomechanics after conventional refractive surgery
}

\author{
Ruirui $\mathrm{Du}^{1}$, Lihua Fang ${ }^{1 *}$, Yinyu Song ${ }^{1}$, Xingming Tao ${ }^{1}$, and Luchao Lin $^{1}$ \\ ${ }^{1}$ Key Laboratory of Nondestructive Test (Ministry of Education), Nanchang Hang kong University, Nanchang, 330063, china
}

\begin{abstract}
Our Purpose is to study the effects of intraocular pressure (IOP) and aspheric transition zone (ATZ) on corneal biomechanics after pure hyperopia correction by using the finite element analysis (FEA). The values of IOP were changed, and 1-5\# aspheric transition zones were designed in 1-5D hyperopia correction model. Simulate and calculate the wavefront aberration, stress and vertex displacement of cornea. The results show that with the increase of IOP and diopter, defocus increases positively and sphere increases negatively. Diopter and IOP have slight influence on coma. At $22 \mathrm{mmHg}$, the maximum value of defocus was $1.367 \mathrm{~mm}$ at $5 \mathrm{D}-1 \mathrm{H}$, and the maximum value of sphere was $-0.32 \mathrm{~mm}$ at $5 \mathrm{D}-5 \#$. IOP and diopter have great influence on the stress in the marginal region of the anterior corneal surface, and 1D-1 \# has the maximum value at $22 \mathrm{mmHg}$. With the increase of IOP and diopter, the vertex displacement of posterior corneal surface increased. The ATZ ablation profile has little effects on the wavefront aberration and displacement. We can draw a conclusion that refractive surgery destroys the physiological structure of cornea and has a great influence on the biomechanical properties of cornea. IOP plays an important role in maintaining the physiological structure of cornea.
\end{abstract}

\section{Introduction}

The cornea was cut in the process of laser in situ keratomileusis (LASIK) refractive surgery. During LASIK refractive surgery, the corneal flap seriously affects the corneal morphology, and the changes of corneal biomechanics will lead to various visual problems after operation. Finite element analysis is a common method to study the biomechanical properties of human eyes, which can solve many problems that can not be completed in vivo experiments, and the results can also provide a strong theoretical basis for clinical ophthalmology.

Intraocular pressure plays an important role in maintaining the stability of corneal biological structure, IOP has individual difference. The influence of IOP on corneal refractive surgery should not be ignored, Elena Lanchares et al [1] have suggested that IOP should be considered in the determination of the depth of ablation. Fang Lihua et al[2] have shown that intraocular pressure can deform the cornea and affect postoperative aberrations. Chen Kaijian et al[3] indicated that high IOP can lead to curvature of the corneal anterior surface, aberrations and refractive changes, and lead to a decline in visual quality. Therefore, IOP is an important factor for postoperative visual quality after refractive surgery.

With the clinical application of corneal refractive surgery, the surgical project is constantly improved. Qvalue guided LASIK is one of the methods. The aspheric transition zone realizes the smooth connection between the optical zone and the non ablation zone. Xin Baoli et al[4-6] said aspheric ablation guided excimer laser corneal refractive surgery improve the vision acuity by decreasing the postoperation wavefront aberration. Lai Xiaohua et al[7] have said that aspheric ablation can improve visual function which make the patient more satisfied, because it maintain the corneal's surface.

Many scholars use the method of finite element simulation to achieve the work that can not be completed in vivo experiment, especially in the field of human eye tissue biomechanics. Ibrahim Seven et al[8] simulated case-specific treatment settings by created the patientspecific finite element models. $\mathrm{R}$ Aldhafeeri[9] constructed the axisymmetric and three-dimensional finite element models of the eye and buckles to calculate eye deformation. Abhijit Sinha Roy et al[10] constructed a finite element whole-eye model of a human eye investigate the impact of corneal elasticity on corneal shape changes before and after simulated LASIK.

\section{Methods and materials}

\subsection{Simulation of LASIK refractive surgery}

Based on the FEA, the LASIK laser refractive surgery was simulated. Firstly, a 3D solid model was built in the 3D modeling software NX, then the material model of

\footnotetext{
Corresponding author: Fanglh71@126.com
} 
human eye tissue was added in the finite element analysis software ANSYS to obtain the finite element model of human eye. then mesh divide, boundary conditions and intraocular pressure were set. In this study, IOP was set to 10,15 and $22 \mathrm{mmHg}$, respectively. The mesh divide of cornea was more detailed than that of sclera, because our research focuses on cornea.

We constructed 1-5D pure hyperopia correction model of human eyes, D is diopter. The thickness of corneal flap was $100 \mathrm{um}$, the diameter of corneal flap surface was $8 \mathrm{~mm}$, the diameter of optical zone was $6 \mathrm{~mm}$, and the width of transition zone was $1.05 \mathrm{~mm}$. According to the nonlinear material properties of cornea and sclera, we use the hyperelastic material model based on Ogden function. The strain energy function can be expressed as:

$$
Q=\sum_{k=1}^{N} \frac{1}{d_{k}}(J-1)^{2 k}+\sum_{i=1}^{N} \frac{\mu_{i}}{\alpha_{i}}\left(\bar{\rho}_{1}^{\alpha_{i}}+\bar{\rho}_{2}^{\alpha_{i}}+\bar{\rho}_{3}^{\alpha_{i}}-3\right)
$$

$N, \mu_{i}, \alpha_{i}$, and $d_{k}$ were the material constants. In the process of material fitting, we choose $N=2$ and $N=$ 1 as the fitting order of cornea and sclera.

Table 1. The corneal and scleral fitting parameters

\begin{tabular}{|c|c|c|c|}
\hline \multicolumn{2}{|c|}{ cornea } & \multicolumn{2}{c|}{ sclera } \\
\hline$\alpha_{1}$ & 103.51 & \multirow{2}{*}{$\alpha_{1}$} & 182.73 \\
\hline$\alpha_{2}$ & 103.61 & & \\
\hline$\mu_{1}$ & $0.003535 \mathrm{MPa}$ & \multirow{2}{*}{$\mu_{1}$} & $0.030224 \mathrm{MPa}$ \\
\hline$\mu_{2}$ & $0.003535 \mathrm{MPa}$ & & \\
\hline
\end{tabular}

\subsection{Aspheric transition zone ablation profiles}

In this study, 1-5\# aspheric transition zone ablation profiles were designed in the 1-5D hyperopia model. The ablation profile of the aspheric transition zone was defined as:

$$
d(x, y)=f_{b}(x, y) \cdot d_{e}(x, y)
$$

And,

$$
d_{e}(x, y)=f\left(\frac{O_{z} x}{2 \sqrt{x^{2}+y^{2}}}, \frac{O_{z} y}{2 \sqrt{x^{2}+y^{2}}}\right)
$$

It defines the extended ablation depth of the transition zone and $O_{z}$ is the diameter of the optical zone.

In addition, $f_{b}(x, y)$ is the mixed function of aspheric transition region algorithm. Before realizing the mixing function, the following aspheric ablation functions must be created:

$$
f_{b}^{\prime}(x, y)=h-\frac{\left(x^{2}+y^{2}\right)}{R+\left[R^{2}-\left(x^{2}+y^{2}\right)(Q+1)\right]^{1 / 2}}
$$

Here, $h$ is the non-zero value of the ablation depth at the edge of the optical zone, $Q$ describe the asphericity. $R$ is the radius of curvature of the ablation profile.

$$
R=\frac{\left(x_{0}\right)^{2}+h^{2}(1+Q)}{2 h}
$$

Then, the aspheric mixing equation can be obtained by normalization method:

$$
f_{b}(x, y)=f_{b}^{\prime}(x, y) / h
$$

Five ablation profiles designed by changing $Q$ value were shown in Figure 1.

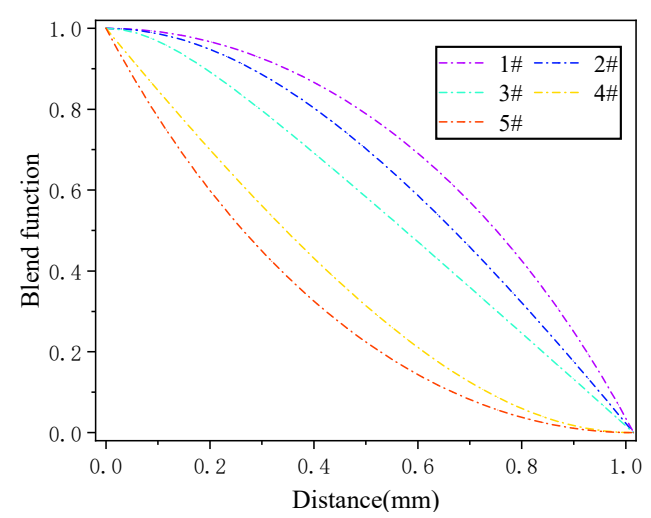

Fig. 1. 1-5\# Ablation profile of aspheric transition zone.

\section{Results}

\subsection{Corneal anterior surface aberrations after hyperopia correction}

In the finite element analysis software, different IOP was set for calculation. Design algorithm based on optical path difference, the wavefront aberrations can be calculated from the $\mathrm{x}-, \mathrm{y}$ - and $\mathrm{z}$-displacements of the anterior corneal surface. Since the wavefront aberrations of the corneal posterior surface is small, it will not be discussed here. Wavefront aberrations were calculated by the difference between postoperative aberrations and preoperative (0D) aberrations. So, we can analyze the optical aberrations caused by biomechanical changes after conventional LASIK refractive surgery.
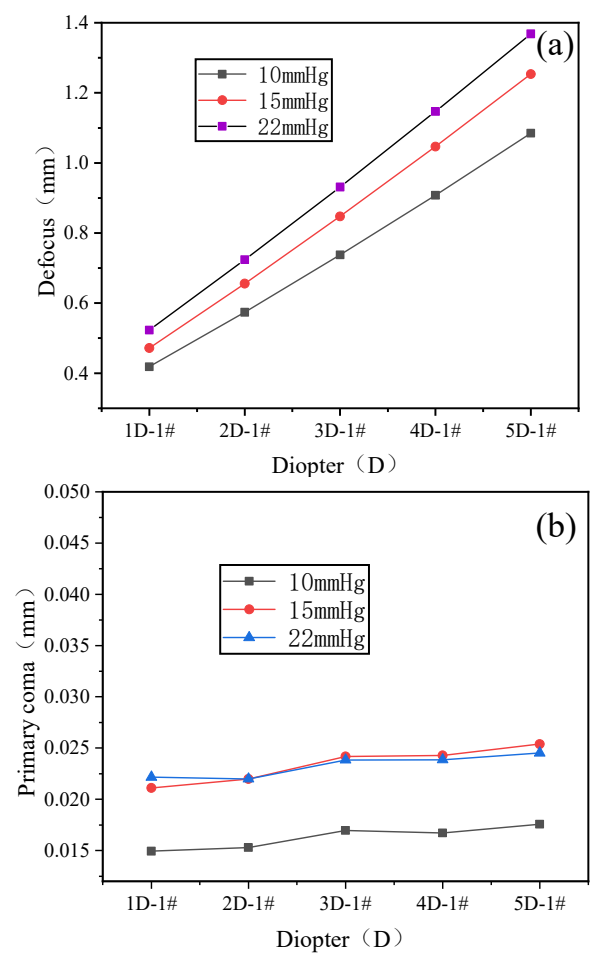

(b) 

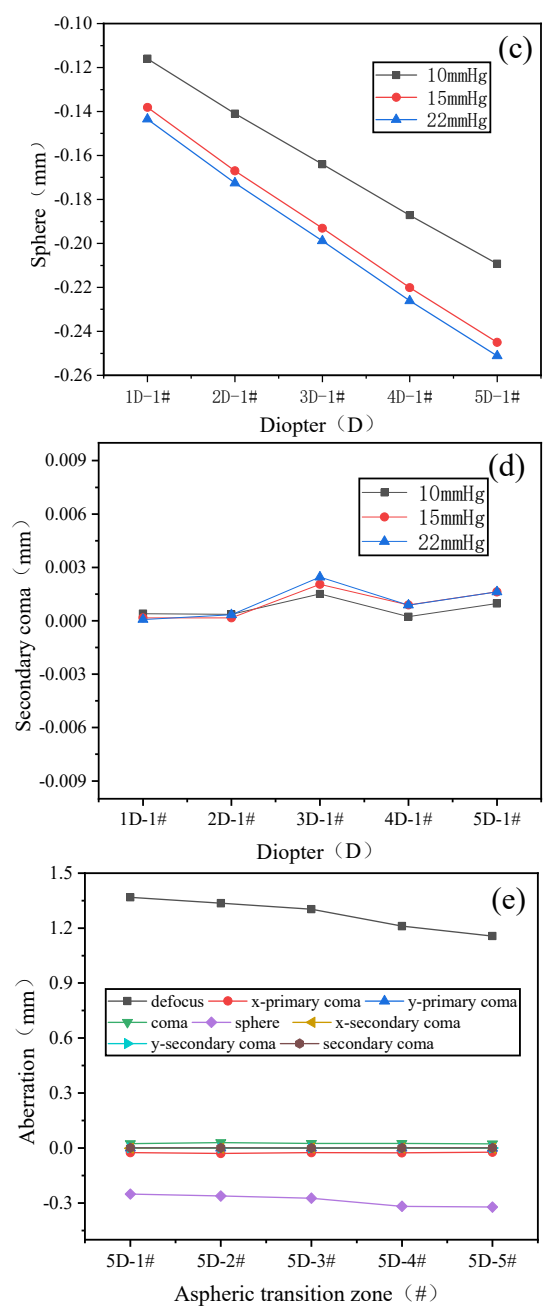

Fig. 2. (a), (b), (c) and (d) represent defocus, primary coma, sphere and secondary coma, respectively. (e) was the aberration induced by different aspheric transition zones under $22 \mathrm{mmHg}$ intraocular pressure.

It can be seen from (a) that IOP and diopter have obvious effects on defocus. Under the same intraocular pressure, the defocus value increased with the increase of hyperopia diopter. At the same diopter, the higher the IOP, the greater the defocus value. At $22 \mathrm{mmHg}, 5 \mathrm{D}-1 \#$ has maximum value of $1.367 \mathrm{~mm}$. From (b), the intraocular pressure has no significant effect on the primary coma, and the value of primary coma at high IOP $(22 \mathrm{mmHg})$ and normal IOP $(15 \mathrm{mmHg})$ have no significant difference. With the increase of corrected diopter, the primary coma has no change, and the value was small as a whole. From (c) we can know that with the increase of intraocular pressure, the sphere increases negatively, but the effect is not obvious. However, under the same intraocular pressure, with the increase of hyperopia diopter, sphere increased rapidly to negative direction. The maximum value was $-0.32 \mathrm{~mm}$ at $5 \mathrm{D}-5 \#$, which can be seen in figure (e). It can be seen from (d) that intraocular pressure and diopter have little effect on the secondary-coma, and the values were small and almost all were $0 \mathrm{~mm}$. From (e), when the intraocular pressure was constant, when the aspheric transition zone changed, defocus and sphere all changed, defocus decreased with the increase of aspheric transition zone ablation profile, but sphere increased in a negative direction. The coma and the secondary coma have no obvious change, and the values of them were almost 0 . In 1-4D hyperopia correction, the effects of different aspheric transition zones on aberration were similar, the figures were not shown here. In addition, the trend at $15 \mathrm{mmHg}$ and $10 \mathrm{mmHg}$ was consistent with that at $22 \mathrm{mmHg}$. On the whole, the IOP was the higher, the defocus and sphere was the greater. The value of 15 $\mathrm{mmHg}$ was not much higher than that of $10 \mathrm{mmHg}$, there was no significant difference. The charts of $15 \mathrm{mmHg}$ and $10 \mathrm{mmHg}$ were not listed here.

\subsection{Stress of corneal anterior surface after hyperopia refractive surgery}

Refractive surgery destroys the integrity of the cornea and the biomechanical properties of cornea were affected. Different cutting depth will produce different value of stress. Here we show the changes of corneal anterior surface stress when the hyperopia correction diopter is 3D.

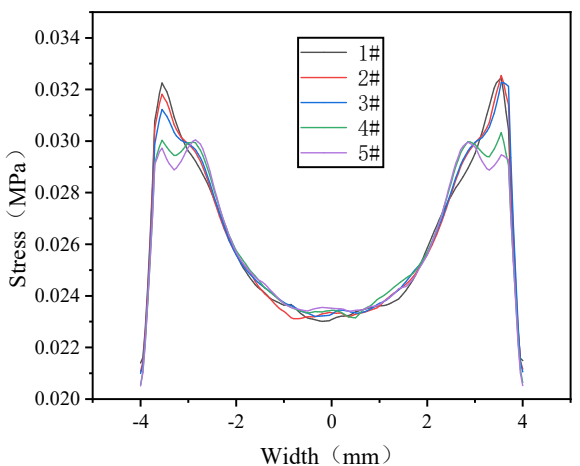

Fig. 3. The corneal anterior surface stress at $22 \mathrm{mmHg}$

It can be seen that 1-5 \# different aspheric transition zones have the same trend with different IOP. The stress value in the central zone is small, the stress value in the edge area is larger, and it is greatly affected by the 1-5 \# aspheric transition zones. This is because that, cutting position in edge area, $1 \#$ aspheric transition zone has the largest cutting amount in the edge region, it also has the greatest influence on the biological structure of cornea. At abscissa 3 and - 3, the attachment produces the maximum value, which is caused by the corneal flap incision. The trend of IOP at 10 and $15 \mathrm{mmHg}$ was the same as that at $22 \mathrm{mmHg}$, but the larger the IOP, the greater the stress. The change of 1D-5D is similar, and the images were not be shown here.

\subsection{The vertex displacement of the posterior corneal surface}

Under the action of intraocular pressure, the anterior and posterior surface of cornea will deform. According to the calculation, the vertex displacement of posterior corneal surface was much larger than that of anterior corneal surface, we will focus on the displacements of the posterior corneal surface. The vertex displacement was calculated by the difference between the maximum value of the anterior and posterior corneal postoperative and preoperative. It can be seen from the calculation that the 
greater the intraocular pressure, the greater the deformation of the anterior and posterior corneal surface.
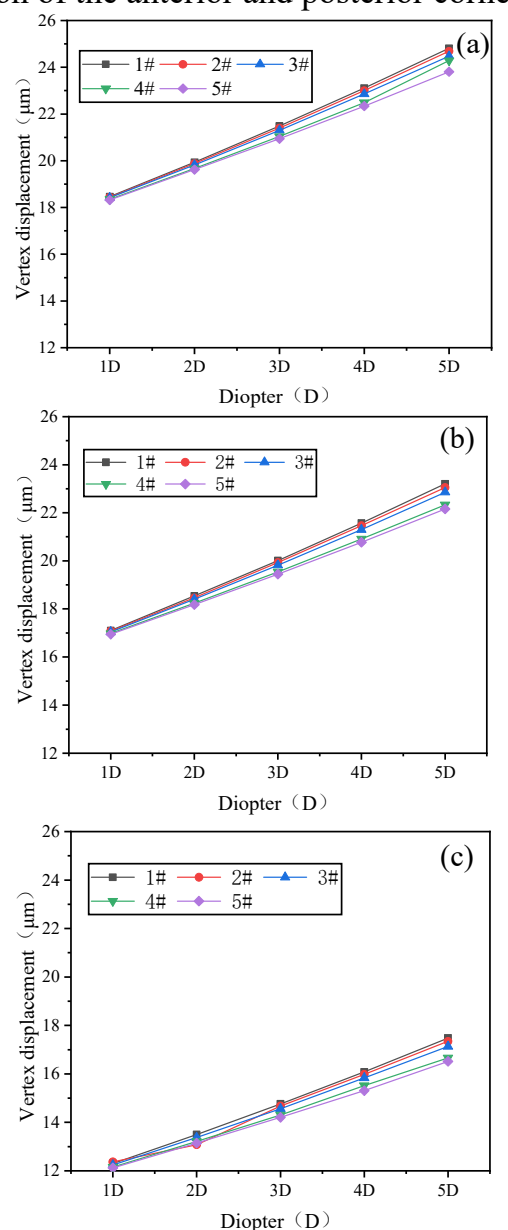

Fig. 4. (a), (b) and (c) showed the displacement of posterior corneal surface when IOP was 22,15 and $10 \mathrm{mmHg}$.

From Figure 4, the vertex displacement was different when the IOP changed. Altogether, the higher the IOP, the greater the deformation of the posterior corneal surface. When the diopter was same, there was no significant difference in corneal deformation under the same IOP, and the deformation of $1 \#-5 \#$ has a slow decreasing trend. When the aspheric transition zone and IOP were same, with the increase of diopter, the deformation was greater. When the IOP was $22 \mathrm{mmHg}$, $5 \mathrm{D}-1$ \# has the maximum value, and it was $24.81 \mu \mathrm{m}$.

\section{Conclusions}

Diopter and IOP have significant influence on defocus and spheren, but have slight influence on coma. With the increase of IOP and diopter, defocus increases positively and sphere increases negatively. IOP and diopter have great influence on the stress in the marginal region of the anterior corneal surface. Due to the cutting amount of the $1 \#$ aspheric transition zone ablation profile, the stress in the $1 \#$ ablation profile was the most concentrated. With the increase of IOP and diopter, the vertex displacement increased. But the aspheric transition zone ablation profile has no obvious effects on the corneal aberration and displacement. IOP[11], diopter and value of $\mathrm{Q}[12]$ will affect the postoperative visual quality. To achieve the best postoperative effect, it is necessary to damage the corneal biomechanical properties as little as possible.

\section{Acknowledgment}

Supported by Natural National Science Foundation of China (61465010), National Key Research and Development Program of China (2018YFE0115700) and Jiangxi Nature Science Foundation (20192BAB207035). Have no conflicts of interest.

\section{References}

1 E. Lanchares, B. Calvo, M. Buey, J. Cristóbal, and M. Doblaré, The Effect of Intraocular Pressure on the Outcome of Myopic Photorefractive Keratectomy: A Numerical Approach[J], J Healthc Eng. 1(3):461-476, (2010).

2 L. Fang, W. Ma, Y. Wang, Y. Dai, and Z. Fang, Theoretical Analysis of Wave-Front Aberrations Induced from Conventional Laser Refractive Surgery in a Biomechanical Finite Element Model[J], Invest Ophth Vis Sci. 61(5):34, (2020).

3 K. Chen, L. Liu, B. Ji, G. Zhang, and L. Tao, The influence of high intraocular pressure on quality of vision after excimer laser corneal refractive surgery[J], J Optom. 16(1):15-19, (2014).

4 B. L. Xin, S. B. Liu, and X. L. Nie, Q-value guided customized laser in situ keratomileusis for the correction of myopia[J], Chinese J Ocular Trauma Occu Eye Disease(With Ophth Surg). 32(4):273-275, (2010).

5 S. Zarei-Ghanavati, H. Gharaee, D. R. Hamilton, J. P. Sanchez, and M. Abrishami, Aspheric versus wavefront-guided photorefractive keratectomy: Contralateral eye study[J], J Cataract Refr Surg. 41(7):1441-1447, (2015).

6 F. Cai and L. Bai, Short-term outcome of aspheric ablation excimer laser corneal refractive surgery[J], Chinese Ophth Res. 26(8):617-620, (2008).

7 X. H. Lai, Q. S. Zhang, X. L. Xie, and X. Jiang, Observation of aspheric ablation in LASIK treating high astigmatism[J], J Clin Ophth. 017(005):401403, (2009).

8 S. Ibrahim, V. Ali, D. Silbiger, R. R. Krueger, and W. J. Dupps, Comparison of Patient-Specific Computational Modeling Predictions and Clinical Outcomes of LASIK for Myopia[J], Invest Ophth Vis Sci. 57(14):6287-6297, (2016).

9 R. Aldhafeeri, Analysis of Scleral Buckling Surgery: Biomechanical Model[D], Univ Pittsburgh, (2017).

10 A. S. Roy and W. J. Dupps, Effects of altered corneal stiffness on native and postoperative LASIK corneal biomechanical behavior: A whole-eye finite element analysis[J], J Refract Surg. 25(10):875, (2009). 
11 L. Hu, Q. Wang, P. Yu, Y. Yu, and D. Zhang, The Influence of Intraocular Pressure on Wavefront Aberrations in Patients Undergoing Laser-Assisted In Situ Keratomileusis, Invest Ophth Vis Sci. 54(8):5527-5534, (2013).

12 J. L. Guo and Q. Wang, Research of the changes of Q-value of the cornea in myopic eyes undertaken excimer laser refractive surgery[J], Med Innov China. 8(04):1-3, (2011). 